Extremity/Lymphedema Image

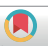

\section{Independent finger-mounted device for vein visualization}

Elijah Zhengyang Cai ${ }^{1}$, Raymond Sui Ming Hon ${ }^{2,3}$, Wai Tung Chow ${ }^{3}$, Ching-Chiuan Yen ${ }^{2,3}$, Thiam Chye Lim ${ }^{1,4}$

${ }^{1}$ Division of Plastic, Reconstructive and Aesthetic Surgery, National University Hospital, Singapore; ${ }^{2}$ Division of Industrial Design and ${ }^{3}$ Keio-NUS CUTE Center, National University of Singapore, Singapore; ${ }^{4}$ Department of Surgery, Yong Loo Lin School of Medicine, National University of Singapore, Singapore

Correspondence: Thiam Chye Lim

Division of Plastic, Reconstructive and Aesthetic Surgery, National University Hospital, Tower Block, Level 8, 1E Kent Ridge Road, Singapore 119228, Singapore

Tel: +65-6-7725083, Fax:+65-6-7778427,E-mail: surlimtc@nus.edu.sg

This research was supported by the National Research Foundation, Singapore under its International Research Centres in the Singapore Funding Initiative and was supported by the National University Health System Clinician Scientist Program-Residency Grant Award 2017.

The authors hold patent rights to the device described in the study-Patent Cooperation Treaty (International application No. PCT/SG2019/050484).

This article was presented at the Asia Pacific Medical Education Conference 2020, on January 8-12, 2020, in Singapore.

We would like to thank Mr. Lau Jo-Hanan (Keio-NUS CUTE Center, National University of Singapore, Singapore) for his effort in assisting with the process of prototyping.

Received: October 26, 2020 • Revised: January 28, 2021

Accepted: February 24, 2021

pISSN: 2234-6163 • eISSN: 2234-617

https://doi.org/10.5999/aps.2020.02096• Arch Plast Surg 2021;48:336-337

Copyright (C) 2021 The Korean Society of Plastic and Reconstructive Surgeons

This is an Open Access article distributed under the terms of the Creative Commons

Attribution Non-Commercial License (https://creativecommons.org/licenses/by-nc/4.0/)

which permits unrestricted non-commercial use, distribution, and reproduction in any

medium, provided the original work is properly cited.

Vein visualization during the harvest of vein grafts, flap elevation, and lymphovenous anastomosis formation can be made more efficient with the aid of a vein visualizer. We developed an independent finger-mounted device (IFMD) based on the concept of transillumination using pen-torches, as we have previously described [1]. This study was approved by our institutional review board. Transillumination works based on the differential light absorption of hemoglobin. Veins appear as silhouettes among adjacent tissues. Commercial transillumination devices are expensive, provide a limited visualization field, and do not allow effective illumination of surfaces with undulating contours due to their rigid framework [2]. Although near-infrared vein visualizers are effective, they are expensive and bulky

[3]. The IFMD was developed with the aim of providing plastic surgeons with an inexpensive pocket-sized tool to assist in vein visualization. IFMD devices are mounted on the thumb and index finger and are swept across the region of interest to assess the superficial venous system (Fig. 1). Individual light sources constructed out of flexible silicone mounted on the fingers function comfortably as a natural extension of the user's fingers. The device measures only $3 \times 2 \mathrm{~cm}$. The independent light sources are multidirectional and easily manipulated in three-dimensional space, catering to variations in contour and skin thickness. Light sources are directed to emerge beneath the veins, forming prominent silhouettes. Veins are identified in real time. The visualization field is increased by increasing the number of devices mounted (Fig. 2). The IFDM is cheap and easy to manufacture. It can be made easily accessible in resource-poor regions. Commercial vein visualizers are more expensive by up to a factor of 10 to 100 .

\section{Notes}

\section{Conflict of interest}

No potential conflict of interest relevant to this article was reported.

\section{Ethical approval}

The study was approved by the Institutional Review

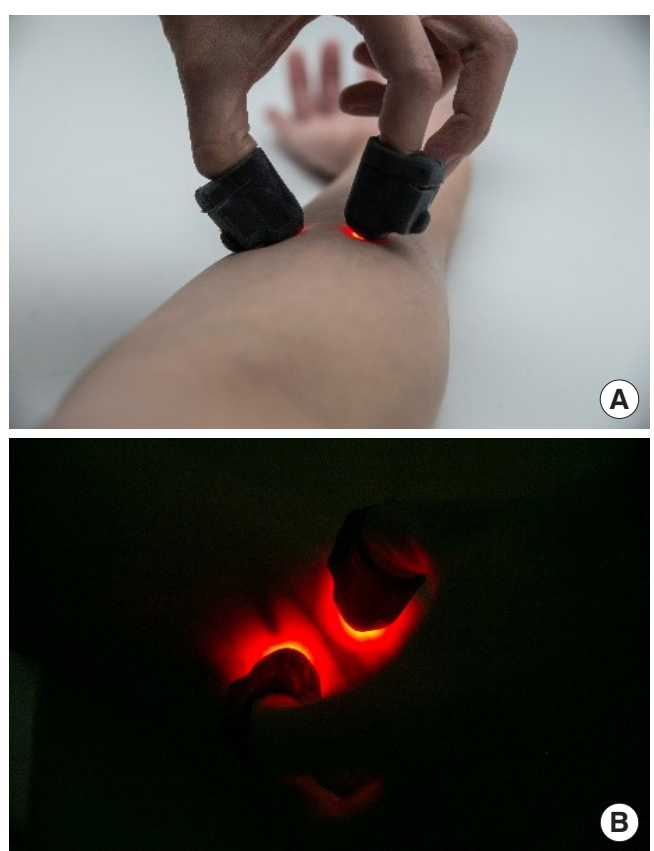

Fig. 1.

The independent fingermounted device. (A) Individual light sources conform to the contours of the forearm, channeling light for optimal vein visualization. (B) Vein over the forearm with branch points, visualized with two finger-mounted devices. 


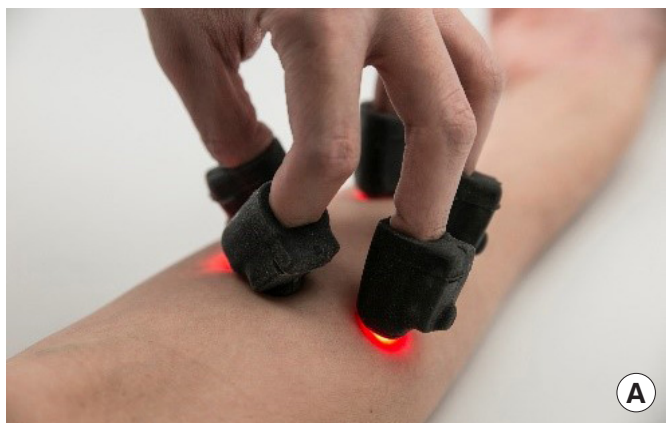

Board of National Healthcare Group, Singapore (IRB No. NHG ROAM DSRB 2018/01266) and performed in accordance with the principles of the Declaration of Helsinki.

\section{Patient consent}

The patients provided written informed consent for the publication and the use of their images.

\section{Author contribution}

Conceptualization, formal analysis, methodology, project administration, and visualization: EZ Cai, RS Hon, WT Chow, CC Yen, TC Lim. Data curation: EZ Cai, TC Lim. Funding acquisition: EZ Cai, TC Lim. Writing - original draft: EZ Cai, RS Hon, WT Chow, CC Yen, TC Lim. Writing - review \& editing: EZ Cai, RS Hon, WT Chow, CC Yen, TC Lim.

\section{ORCID}

Elijah Zhengyang Cai

https://orcid.org/0000-0001-9466-6871

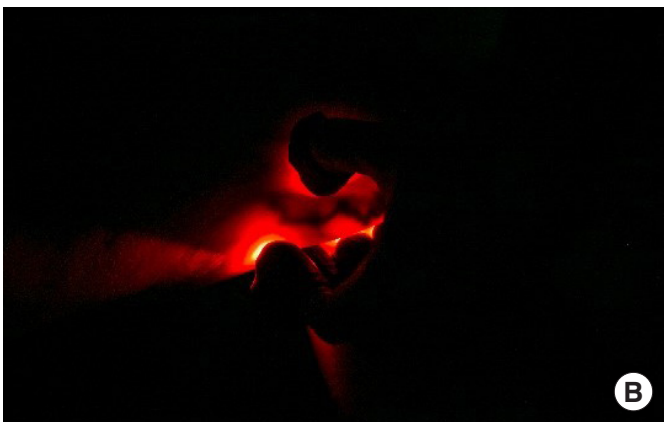

Fig. 2.

Additional application of the independent finger-mounted device. (A) Devices mounted on all digits to expand the field of visualization, an additional benefit of independent finger-mounted light sources. (B) Varicose veins visualized.
Raymond Sui Ming Hon

https://orcid.org/0000-0003-1844-3574

Wai Tung Chow

https://orcid.org/0000-0001-6501-5367

Ching-Chiuan Yen

https://orcid.org/0000-0003-4325-1689

Thiam Chye Lim

https://orcid.org/0000-0001-6084-2902

\section{References}

1. Cai EZ, Sankaran K, Tan M, et al. Pen torch transillumination: difficult venepuncture made easy. World J Surg 2017;41:2401-8.

2. Katsogridakis YL, Seshadri R, Sullivan C, et al. Veinlite transillumination in the pediatric emergency department: a therapeutic interventional trial. Pediatr Emerg Care 2008;24:83-8.

3. Karaaltin MV. Utilizing the vein viewer technology to map out a venous flap preoperatively.J Reconstr Microsurg 2013;29:423-4. 